\title{
The Influence of Characteristics of Thermodynamic Effect and Bulk Viscosity Effect on the Bubble Dynamics
}

\author{
Wenjun Kong ${ }^{1, a}$, Ce Guo ${ }^{1, b}$, Jinming Liao ${ }^{1, c}$ and Xijing Zhu ${ }^{1, d}$ \\ ${ }^{1}$ School of Mechanical and Power Engineering, North University of China, Tauyuan 030051, China \\ akwj2002@163.com, 'buoce1027@163.com, ${ }^{\mathrm{c} l i a 01102044342 @ 163 . c o m, ~}{ }^{\mathrm{d}} \mathrm{ZXJ161501@nuc.edu.}$ \\ $\mathrm{cn}$
}

Keywords: Bulk viscosity, thermodynamic effect, bifurcation chaos, ultrasonic.

\begin{abstract}
The important influence of thermodynamic effect inside bubble and viscous liquid volume outside the bubble on the dynamics of bubble can be found. However, the combined effect of those two factors has currently little research and the thermodynamic effect of the bubble can be seen from the Flynn model. Taking thermodynamic effect inside bubble and viscous liquid volume outside bubble into consideration, the Flynn model can be revised under ultrasonic excitation. The effect of ultrasonic frequency, ultrasonic amplitude and the initial bubble radius on the dynamics of the bubble can be simulated numerically. For comparison with Flynn model, the chaotic and bifurcation diagram of therevised model can be obtained. It can be seen that the thermodynamic effect can extend the time in the stable states, and viscous liquid volume can restrain the random motion of the bubble. To achieve obvious cavitation effect, a higher intensity ultrasonic excitation can be required which the motion of the bubble can be from bifurcation into chaotic. The results solve the problem that the Flynn model not fits the bubble movement well actually. It is provided a theoretical basis for the potential application of the cavitation effect.
\end{abstract}

\section{Introduction}

When ultrasonic wave acts on a liquid, if the frequency and amplitude exceed certain thresholds of liquid, cavitation occurs in the liquid, causing the formation of many bubbles. Bubbles have stronger nonlinear effect and kinetic characteristics than the normal bubbles. Thus, the cavitation bubble dynamics in a liquid has always been considered as a focus field in the ultrasonic application ${ }^{[1-2]}$. At present study, many important factors influcening bubble dynamics such as the viscosity of liquid on the bubble and the vapor pressure from gas inside the bubble, and so on, were ignored for simplication ${ }^{[3-4]}$. However, these factors cannot be neglected in some certain cases, it is necessary to make further researches on them so that the models would precisely describe the moving state of the bubble. Based on Flynn Mode, this paper develops a bubble model considering the bulk viscosity of liquid and the thermodynamic effect of gas inside bubbles, then the influence of exciting acoustic pressure, exciting frequency and initial balance radius on bubble dynamics can be discussed using the theory of bifurcation chaos. Compared with the results of Flynn model, the result revised reveals a different bubble dynamics. In addition, this paper reveals the characteristics of bifurcation and chaos of bubbles vibrated, which provides the theoretical basis for the potential applications of cavitation bubbles.

\section{Model}

In order to study the cavitation bubble dynamics under the effect of acoustic field, the following assumptions were adopted: Cavitation bubble is spherical and only radial motion of bubble is discussed during shrinkage and swelling, The main part of liquid is water and the temperature is constant at $25^{\circ} \mathrm{C}$; The compressibility, bulk viscosity, shear viscosity, surface tension and vapor pressure of liquid are considered in the model; The heat exchange, the phase change of vapor, the mass exchange of gas and the chemical reaction inside the bubbles are not taken into account. 
The Flynn model takes into account the thermodynamic effect of gas inside bubbles and the energy distribution of liquid around bubbles based on the theoretical deduction ${ }^{[5-6]}$. Consequently, Flynn model provides more information about the changing thermodynamic process. It is given by the following equation:

$$
\begin{aligned}
\left(1-\frac{\dot{R}}{c}\right) R \ddot{R}+\frac{3}{2} \dot{R}^{2}\left(1+\frac{\dot{R}}{3 c}\right)= & \left(1+\frac{\dot{R}}{c}\right) \frac{1}{\rho}[P(R, t)-P(\infty, t)] \\
& +\frac{R}{\rho c}(1-\dot{R} / c) \frac{d[P(R, t)-P(\infty, t)]}{d t}
\end{aligned}
$$

where, $P(\infty, t)=P_{0}+P_{A} \sin (2 \pi f t)$ and $P_{0}$ is the ambient atmospheric pressure, $P_{A}$ is the amplitude of exciting acoustic waves is $\rho$ is the density of liquid, and $R$ is the radius of bubbles at random time, $\dot{R}$ is the change ratio of radius of bubbles, $\ddot{R}$ is the change ratio of fluctuating velocity, $c$ is the propagation velocity of acoustic waves in liquid, and $P(R, t)$ is the pressure of liquid surface of bubbles.

Due to the continuity of pressure and normal velocity for the superficial liquid and gas, the expression of $P(R, t)$ can be expressed as

$$
P(R, t)=P_{g}(R, t)+P_{v}-2 \sigma / R-4 \mu_{s} \dot{R} / R
$$

where, $P_{g}=\left(P_{0}+\frac{2 \sigma}{R}-P_{v}\right)\left(\frac{R_{0}}{R}\right)^{3 \kappa}$ is the pressure on the internal surface of bubbles, $\kappa$ is the polytropic index for ideal gas, $P_{v}$ is the saturated vapor pressure inside bubble, $\sigma$ is the liquid surface tension coefficient, and $\mu_{s}$ is the liquid shear viscosity coefficient.

Assuming that the oscillating thermal process of gas in bubbles is independent of the compressibility process of gas outside bubbles, the amended thermal oscillating process is introduced based on Flynn Model. Thus the poly tropic equations of ideal gas can be revised as follows[7]:

$$
\left(4 \pi R^{2} c d t\right)^{\kappa} P_{g}=\left[4 \pi R^{2}(c+\dot{R}) d t\right]^{\kappa} P_{g}^{i n}
$$

where, $P_{g}^{\text {in }}$ is the amended pressure on the inside bubble walls, and it can be expressed:

$$
P_{g}^{i n}=(1+\dot{R} / c)^{-\kappa} P_{g}
$$

The shear viscous effect in Eq. (1) is taken into consideration, but the bulk viscous effect is ignored. However, in fact, the bulk coefficient of viscosity and the shear viscosity coefficient are in the same order of magnitude generally, and even in some cases, the influence of bulk coefficient of viscosity on bubble dynamics is more obvious. According to the theory of the fluid mechanics, the relations of the viscous force $P_{\mathrm{N}}$ on bubbles caused by fluid and the shear viscosity coefficient $\mu_{\mathrm{s}}$ can be expressed as:

$$
P_{N}=-\mu_{s}\left(2 \frac{\partial u}{\partial r}\right)-\left(\mu_{b}-\frac{2}{3} \mu_{s}\right)\left(\frac{\partial u}{\partial r}+\frac{2 u}{r}\right)
$$

where, $u$ is the radial motion speed of the bubbles, $r$ is the radial movement distance. In practical situations, the shear viscosity coefficient $\mu_{s}$ and bulk viscosity coefficient $\mu_{b}$ change with the change of temperature, and it can expressed as:

$$
\begin{gathered}
\mu_{s}=1.008 \exp \left[\frac{2.41(20-T)+0.0054(T-20)^{2}}{79.65+T}\right] \\
\mu_{b}=\frac{2 \alpha \rho v^{3}}{\omega^{2}}+\frac{4 \mu_{s}}{3}+\frac{(\gamma-1) \tau}{C_{p}}
\end{gathered}
$$

where, $T$ is the temperature, $\alpha$ is the attenuation coefficient, $v$ is the speed, $\gamma$ is the heat capacity ratio, $\tau$ is the coefficient of thermal conductivity, $C_{p}$ is the specific heat at constant pressure. 
In Eq. (14), it is obvious that the calculation of $\mu_{b}$ is complicated. So, it can be find out according to this empirical formula in practical cases:

$$
\mu_{b}=B_{0}+B_{1} T+B_{2} T^{2}+B_{3} T^{3}
$$

wherer, the coefficient $B_{0}, B_{1}, B_{2}, B_{3}$, of this expression can be described as:

$$
B_{0}=5.94068 \times 10^{-3}, B_{1}=-2.34073 \times 10^{-4}, B_{2}=4.94789 \times 10^{-6}, B_{3}=-3.97502 \times 10^{-8}
$$

Therefore, taking the Eq. (3)-(9) into the Eq. (2), the pressure of liquid surface of bubbles $P(R, t)$ can be revised as:

$$
P(R, t)=(1+\dot{R} / c)^{-\kappa} P_{g 0}\left(\frac{R_{0}}{R}\right)^{3 \kappa}+P_{v}-\frac{2 \sigma}{R}-4 \mu_{s} \frac{\dot{R}}{R}-\frac{1}{\rho c^{2}}\left(\frac{4}{3} \mu_{s}+\mu_{b}\right) \frac{d P}{d t}
$$

Combination the Eq. (1) with the Eq. (10), the revised Flynn model considering the process of thermodynamic changes inside the bubbles and the bulk viscosity of liquid can be developed.

\section{Simulation calculation}

The fourth order Runge-Kutta's method is used to solve the revised model Eq. (1). The initial conditions of the bubble system can be shown as $R(t=0)=R_{0}, d R / d t(t=0)=0, \theta(t=0)=0$. Assuming that the movement of bubbles is the isothermal process, namely $\kappa=1$, using water as the fluid medium, the physical parameters are $\rho=998 \mathrm{~kg} / \mathrm{m}^{3}, \quad \sigma=0.0725 \mathrm{~N} / \mathrm{m}, \quad P_{0}=1 \times 10^{5} \mathrm{~Pa}$, $P_{v}=2033 \mathrm{kPa}, c=1500 \mathrm{~m} / \mathrm{s}, \mu_{s}=0.001 \mathrm{~kg} /(\mathrm{m} . \mathrm{s}), \mu_{b}=0.0025 \mathrm{~kg} /(\mathrm{m} . \mathrm{s})$. For the reason that there are three important parameters: the bubble equilibrium radius $R_{0}$, acoustic excitation frequency $f$ and the excitation amplitude of sound wave $P_{A}$. These three parameters have a great influence on the motion state of bubbles, the results of the model depends much on them .The influence of the three parameters on the results of the model will be discussed below.

Acoustic frequency. Setting the initial radius of the bubble as $10 \mu \mathrm{m}$, the amplitude of the incentive acoustic as $2.9 \times 10^{5} \mathrm{~Pa}$, The phase diagram of a cavitation bubble under different frequency is shown in Fig.1. From the Fig. 1(a), as the incentive acoustic frequency continuously increases, the motion state of bubbles transfers from the period1 to the period 2, and finally to the period 4, then entering into the transition of the chaos state. With the further increasing the acoustic frequency, the state of bubbles turns into the period 1,the period 2,and the period 4 ,etc, then enters into the chaos again. This kind of periodic motion and chaotic motion alternates, show that the incentive acoustic frequency has a great influence on the motion states of bubbles. With further increase of frequency of sound wave, reverse bifurcation appears. It can be found out from the diagram that in the range of frequency of $340-410 \mathrm{kHz}$, the motion of bubbles is in a state of chaos, when the acoustic frequency is in this range, the obvious cavitation can be observed in the liquid. The Fig. 1(b) is the bifurcation diagram which shows the relationship between the maximum radius of bubbles $R / R_{0}$ and the incentive acoustic frequency $\mathrm{f}$ in the movement process. From Fig. 1(b), it can be found that when the incentive acoustic frequency is over $240 \mathrm{kHz}$, the bubbles begin to enter into a state of chaos.

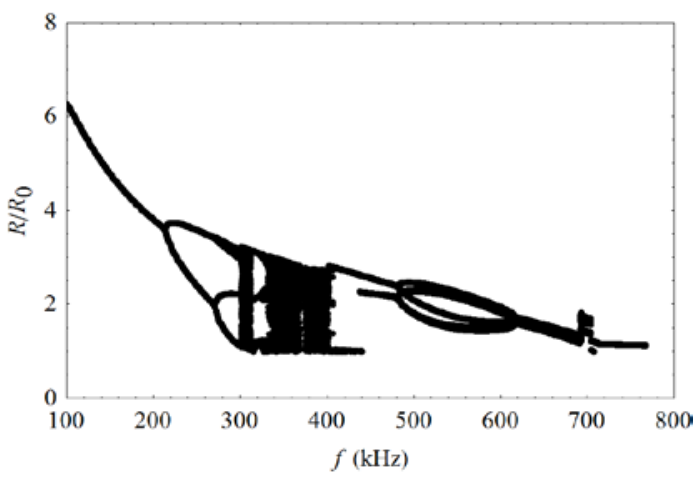

(a) Rvised model

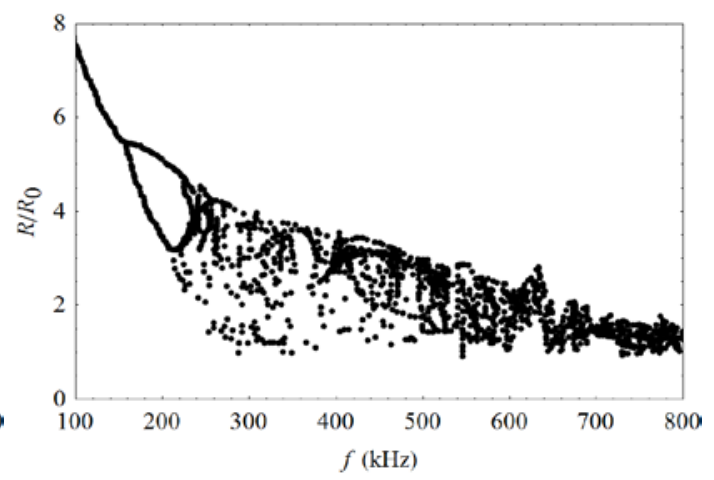

(b) Flynn model 
Fig. 1 The influence of the frequency on the motion state of the bubble

Comparing Fig.1(a) and Fig.1(b), the bubble dynamics is changed and even blocked and the change is appeared in the range of high frequency when considering the bulk viscosity effect of the liquid outside the bubble and the thermodynamic effect of gas inside the bubble.

Acoustic amplitude. From the discussion above, it can be concluded that bulk viscous effect hindered the random movement of bubbles, and the thermodynamics effect of gas inside the bubble weakened the random movement of the bubbles under the influence of a high frequency sound wave excitation. The maximum of radius of bubbles $R / R_{0}$ versus the incentive acoustic amplitude value is shown in Fig.2. It can be found from Fig.2 (a) when the acoustic amplitude value is over $250 \mathrm{kPa}$, the bubbles begin to perform irregular motion, which is obvious cavitation. From Fig.2 (b), it is clear that the bubbles begin to be in the state of chaos when the amplitude of the incentive acoustic is more than $210 \mathrm{kPa}$. According to the comparison between the results of the Fig.2 (a) and the Fig.2 (b), on the same initial conditions, when considering the bulk viscosity effect of the liquid outside the bubble and the thermodynamic effect of gas inside the bubble, the system requires higher amplitude of incentive sound wave to enter into the state of chaos. As a result, to obtain obvious cavitations among liquid, it needs to increase the excitation frequency and acoustic amplitude. However, in our research, the bubble dynamic is calculated at $R_{0}=10 \mu \mathrm{m}$ and in reality liquid, the physical characteristics of the bubble in itself and the environment both have a great influence on the results, so the effect of the initial bubble radius on the characteristics of bubbles should also be considered.

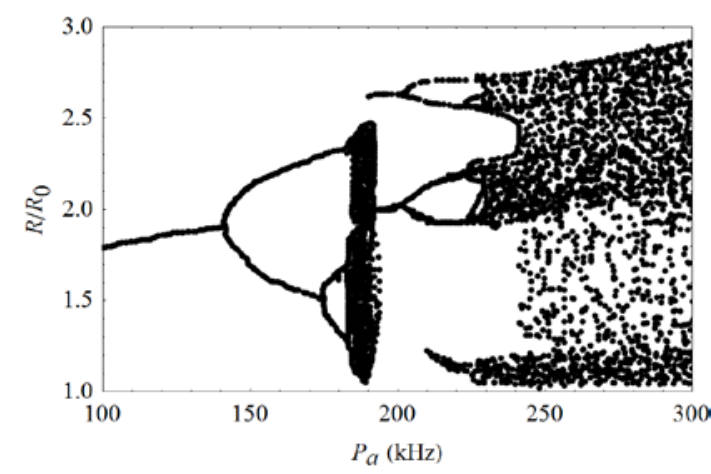

(a) Revised model

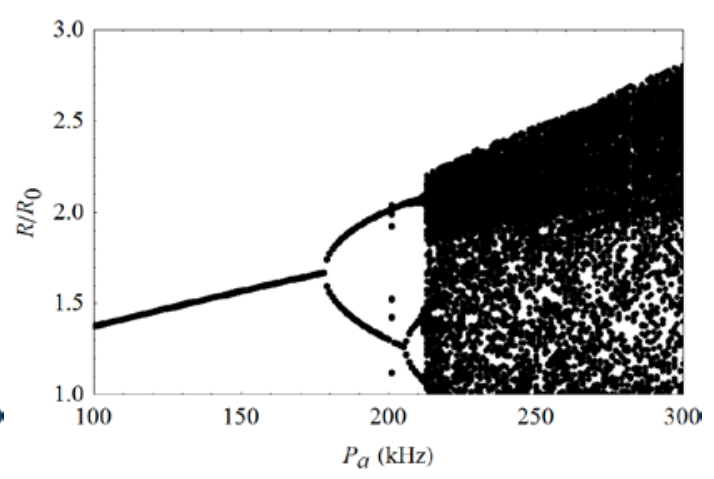

(b) Flynn model

Fig. 2 The influence of the acoustic amplitude on the motion state of the bubble

Initial radius of bubble. The change of the maximum radius of bubbles in the process with the initial radius $R_{0}$ is shown in the Fig. 3. It can be seen that with the increase of initial radius of the bubbles, the relation between the largest radius and the initial radius is linear from Fig. 3(a). It shows that only the environment is transformed to realize the larger initial radius. Obvious cavitation will happen in the liquid. However, in the experiment, it can be achieved by changing the type of liquid and the liquid temperature, and actually the results of the experiment are smaller than the theoretical value. It is because in the process of increasing the size of bubbles, the thermodynamic effect of gas inside the bubble and the bulk viscosity effect of the liquid outside the bubble have a great influence on bubble movement status, while these factors are not considered in the Flynn model. As a result, the way of the motion state of the bubbles changes will be discussed. The bifurcation diagram of the change of the maximum radius $R / R_{0}$ in the motion process of bubbles with the change of the initial radius $R_{0}$ is shown in Fig.3 (b). From Fig.3 (b), the bifurcation effect is not obvious, which means the initial radius has a complicated influence on the initial motion state. But it is obvious that when the initial radius is small, the bubble motion in the process of maximum radius is large, and cavitation effect is obvious, when the initial radius of bubble becomes large, the radius in the process of bubble motion is small.

With comparison between the Fig.3 (a) and the Fig.3 (b), it can concluded that when the initial radius is small ,the simulation in the Flynn model and the modified model have the same result, the maximum radius of bubbles in the movement process has the same order of magnitude, but when the initial radius is larger, the maximum radius of the bubbles in the modified model is obviously shorter 
than that in Flynn model, it cannot be ignored, it is significant in practical application, and it is worth further discussing.

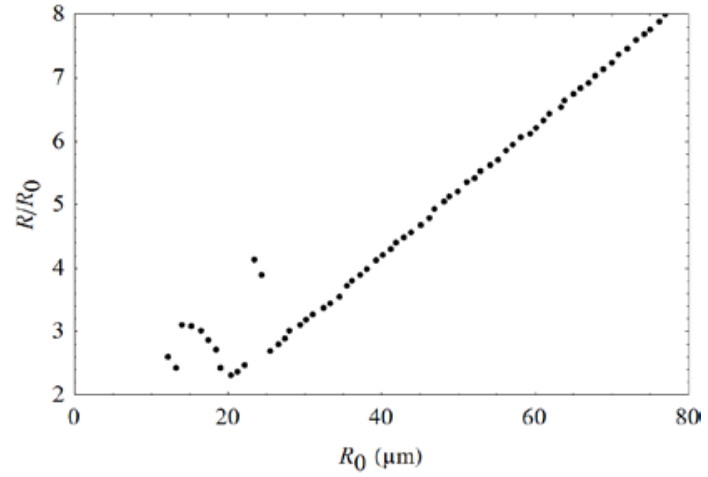

(a) Revised model

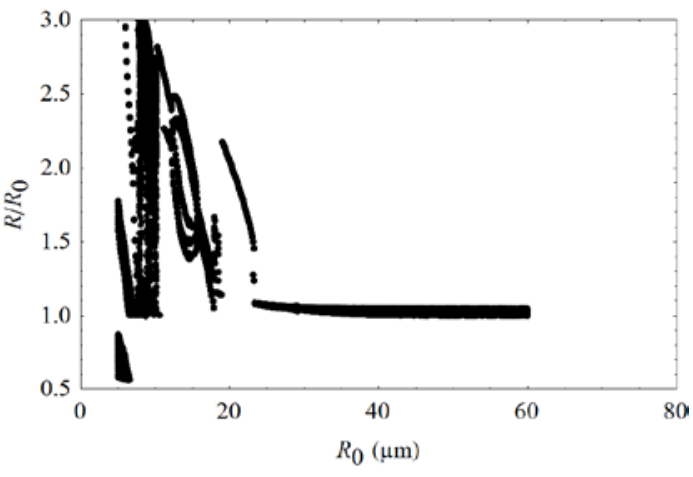

(b) Flynn model

Fig. 3 The influence of the initial radius on the motion state of the bubble

\section{Summary}

A revised bubble model based on Flynn model which considers the thermodynamics process of gas inside the bubble is developed. The numerical results show that when cavitation happens in the liquid, the acoustic frequency and amplitude threshold required are higher than Flynn model, and when the initial radius is larger, the cavitation effect is not as obvious as that in Flynn model. Thus, it is required a higher frequency and acoustic amplitude to stimulate nonlinear bubble dynamics and produce obvious cavitation. As is expected in this paper, the modified model is consistent with the actual experimental results basically. However this article is based on the study of a single bubble and the bubble dynamics is the assumed strictly, and the results of the model cannot be directly applied to a condition where many bubbles exist, so more in-depth and extensive theoretical researches are needed in this area.

\section{Acknowledgements}

This work was financially supported by the National Natural Science Foundation of China (50975265 and 51275490), Shanxi Province Natural Science Foundation (2013011024-5) and Graduate Innovation Foundation of Shanxi Province of China (Grant No. 20143077)

\section{References}

[1] Zhu Xi Jing, Guo Ce, Wang Jian Qing, Liu Guo Dong, Dynamics modeling of cavitation bubble in the grinding area of power ultrasonic honing, Advanced Materials Research, 797 (2013) 108-111.

[2] M.Mahdi, R.Ebrahimi, M. Shams, Numerical analysis of the effects of radiation heat transfer and ionization energy loss on the cavitation bubble’s dynamics, Physics Letters A, 375 (2011)2348-2361.

[3] Smeraka P, Birnir B, Banerjee S, Regular and chaotic bubble oscillations in periodically driven pressure fields, Physics of Fluids,30(1987) 3342-3350.

[4] Javier J F, Chaotic gas bubble oscillations in a viscoelas-tic fluid, Comptes Rendus Mécanique, 5(2008) 411-416.

[5] Flynn HG, Cavitation dynamics, I. A mathematical form-utation, J Acoust Soc Am,6(1975) 1379-1396.

[6] Keller JB, Miksis M, Bubble oscillations of large amplitude, J Acoust Soc Ann, 2(1980) 628-633.

[7] Jimenez-Fernandez J, Crespo A, Bubble oscillation and inertial cavitation in viscoelastic fluids, Ultrasonics, 8(2005) 643-651. 
